\title{
Neutron scattering study of the layered Ising magnet $\mathrm{CsDy}\left(\mathrm{MoO}_{4}\right)_{2}$
}

\author{
E.N. Khatsko \\ B. Verkin Institute for Low Temperatures Physics and Engineering of the National Academy \\ of Sciences of Ukraine, 47 Lenin Ave., Kharkov 61103, Ukraine \\ E-mail: khatsko@ilt.kharkov.ua
}

\section{A. Zheludev ${ }^{\star}$}

Physics Department, Brookhaven National Laboratory, Upton, NY 11973-5000, USA

\section{J.M. Tranquada}

Physics Department, Brookhaven National Laboratory, Upton, NY 11973-5000, USA

\section{W.T. Klooster ${ }^{* \star}$}

Chemistry Department, Brookhaven National Laboratory, Upton, NY 11973-5000, USA

\author{
A.M. Knigavko \\ Department of Physics, University of Alberta, Edmonton, Alberta, T6G $2 J 1$ Canada \\ R.C. Srivastava \\ Department of Physics, Indian Institute of Technology, Kanpur 208016, India
}

Received June 18, 2003, revised August 13, 2003

\begin{abstract}
The quasi-two-dimensional antiferromagnet $\operatorname{CsDy}\left(\mathrm{MoO}_{4}\right)_{2}$ is studied by neutron diffraction and quasielastic neutron scattering. The crystal structure of two low-temperature phases (below $120 \mathrm{~K}$ and below $40 \mathrm{~K}$ ) is determined. An approximate structure of the magnetically ordered phase $\left(T_{N}=1.36 \mathrm{~K}\right)$ is proposed. In the ordered state the order-parameter critical exponent $\beta=0.17(0.01)$, the in-plane correlation length exponent $v=0.94(0.07)$, and the staggered susceptibility critical index $\gamma=1.01(0.04)$ were determined. Comparing these results to the exact solution for a 2D Ising magnet we conclude that, although 2D behavior is apparent in $\mathrm{CsDy}\left(\mathrm{MoO}_{4}\right)_{2}$, there are deviations from the simple 2D Ising model.
\end{abstract}

PACS: 61.12.-q

\section{Introduction}

For many years low-dimensional (low-D) magnetism has remained at the forefront of solid-state research. Experimental studies of magnetic phase transitions in quasi-low-D compounds are very important for the general understanding of critical phenomena. The study of 2D Ising compounds is of special interest, since for certain low-dimensional Ising theoretical models there is an exact solution and the results can be directly compared to experiment. A relatively few ma-

* $\quad$ Present address: Solid State Division, Oak Ridge National Laboratory, Oak Ridge, TN 37830, USA.

** Present address: Bragg Institute, Building 58, Australian Nuclear Science and Technology Organisation, PMB 1, Menai NSW 2234, Australia 
terials simultaneously have strong single-ion anisotropy and adequate two-dimensional magnetic interactions to qualify as model 2D Ising magnets.

Double rare-earth molybdates (DRMs) with the general formula $\mathrm{MR}\left(\mathrm{MoO}_{4}\right)_{2}(\mathrm{R}=$ rare earth, $\mathrm{M}=$ alkali metal) crystallize in a variety of layered structures (see for example Refs. 1-4) and are a large family of $2 \mathrm{D}$ compounds with some very interesting species. The magnetism of molybdates is entirely due to the presence of trivalent rare-earth ions. For the latter, magnetic anisotropy effects are crucial, and components of the $g$ tensor may vary from almost 0 to as much as 20. In many DRMs the rare-earth sites behave as nearly ideal Ising centers. Typically large rare-earth magnetic moments in many cases result in appreciable dipolar magnetic interactions $[5,6]$. Long-range magnetic order has been detected in several species [7-9] and typically occurs only at sub-Kelvin temperatures. The rather unusual magnetic properties of DRMs have been investigated using dc and ac magnetic measurements [7-9], optical experiments [10,11] and ESR [12,13].

Although neutron diffraction has been widely used in the study of crystal structures $[1,3,14]$, we were surprised not to find any reports in the literature on magnetic neutron scattering experiments on DRMs. Although magnetic neutron diffraction often is the «ultimate» technique for studying magnetic phase transitions and critical behavior, actual experiments may be, from a purely technical point of view, nontrivial to carry out. Low magnetic ordering temperatures require the use of bulky cryogenic equipment that limits the region of $Q$ space accessible in a diffraction experiment. In addition, most DRMs go through a whole series of structural transformations when cooled down from room temperature, and the formation of crystallographic domains may complicate the interpretation of the diffraction pattern. These problems indeed have to be dealt with, but, as we show below, do not present an insurmountable obstacle.

In the present paper we report the results of neutron scattering experiments on one particular compound, namely $\mathrm{CsDy}\left(\mathrm{MoO}_{4}\right)_{2}$. Data pertaining to the crystallographic and magnetic structures, as well as measurements of the magnetic critical behavior, confirm the 2D Ising nature of this system. At the same time, certain new features hint at the important role played by dipolar interactions.

\section{Experimental}

Our choice of $\mathrm{CsDy}\left(\mathrm{MoO}_{4}\right)_{2}$ over other DRM species was largely governed by the availability of large single-crystal samples. Among the rare-earth ions, $\mathrm{Dy}^{3+}$ in the molybdates has the advantage of having one of the largest magnetic moments. The tradeoff is its appreciable neutron absorption cross section. $\mathrm{CsDy}\left(\mathrm{MoO}_{4}\right)_{2}$ would not be an ideal candidate for inelastic experiments, but is well-suited for measurements of elastic magnetic scattering. Mica-like transparent rectangular single-crystal samples for our experiments were prepared by spontaneous crystallization from melt solution [15]. $4 \times 4 \times 0.15 \mathrm{~mm}$ and $8 \times 12 \times 0.15 \mathrm{~mm}$ samples were used for conventional and magnetic neutron scattering experiments, respectively.

4-circle diffraction data were collected at the H6M 4-circle diffractometer at the High Flux Beam Reactor (HFBR) at Brookhaven National Laboratory. In this experiment the sample environment was a standard Displex refrigerator. Two separate sets of $(h k l)$ data were collected at $50 \mathrm{~K}$ and $15 \mathrm{~K}$, respectively.

Low-temperature $(0.35-5 \mathrm{~K})$ neutron scattering experiments were performed at the $\mathrm{H} 8, \mathrm{H} 7$, and $\mathrm{H} 4 \mathrm{M}$ 3 -axis spectrometers at the HFBR with the use of a pumped- ${ }^{3}$ He Dewar. The temperature was controlled with an automated resistance bridge to a precision of $\approx 0.01 \mathrm{~K}$. The sample was wrapped in thin aluminum foil and mounted as strain-free as possible. A neutron beam of fixed final energy $E_{f}=14.7 \mathrm{meV}$ was used with a pyrolitic graphite (PG) filter positioned after the sample. Pyrolitic graphite (002) reflections were used for the monochromator and analyzer. The collimation setup was $40^{\prime}-40^{\prime}-40^{\prime}-80^{\prime}$. The sample mosaic was found to be of the order of $2.5^{\circ}$ full width at half maximum (FWHM). Special care was taken to precisely measure the dimensions and orientations of the crystallographic faces. This information was later used to analytically calculate absorption corrections to the measured intensities.

\section{Results}

\subsection{Crystal structure}

At room temperature $\mathrm{CsDy}\left(\mathrm{MoO}_{4}\right)_{2}$ was believed to be orthorhombic, space group Pccm, with cell constants $a=9.51 \AA, b=5.05 \AA$, and $c=7.97 \AA$ [3]. This structure is essentially the same as for $\operatorname{Cs} \operatorname{Pr}\left(\mathrm{MoO}_{4}\right)_{2}$ [16]. The most prominent feature is a stacking of alternating layers along the crystallographic $a$-axis: $-\mathrm{Dy}-\left(\mathrm{MoO}_{4}\right)-\mathrm{Cs}-\left(\mathrm{MoO}_{4}\right)-($ Fig. 1,a). Each layer is parallel to the (100) perfect cleavage plane. The arrangement of magnetic ions is quasi-two-dimensional. The interlayer Dy-Dy distance is large, $\approx 9.5 \AA=a$ (Fig. 1,b). Within each layer the magnetic sites form a rectangular lattice with nearest-neighbor distances $\approx 5 \AA=b$ and $\approx 4 \AA=c / 2$. Although the Pccm structure has not been seen in any of our $\mathrm{CsDy}\left(\mathrm{MoO}_{4}\right)_{2}$ samples at any temperature, all the crystallographic phases 


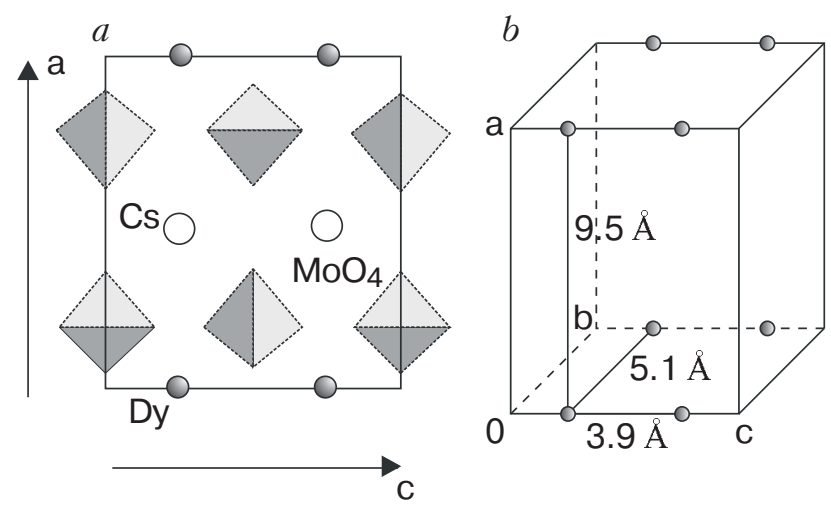

Fig. 1. «Parent» model of the Pccm structure of $\mathrm{CsDy}\left(\mathrm{MoO}_{4}\right)_{2}$. (a) A projection onto the ac crystallographic plane showing the Dy and Cs sites as well as the $\mathrm{MoO}_{4}$ tetrahedra. (b) Structural relation between nearest-neighbor magnetic $\mathrm{Dy}^{3+}$ ions in the unit cell.

that were observed (see below) can be described as distortions of this «parent» structure.

For our samples the room-temperature structure was found to be monoclinic, with the measured cell parameters $a=9.49 \AA, b=5.05 \AA, c=7.97 \AA$ and $\beta=88.4^{\circ}$. The two monoclinic domains share common $a^{*}$ and $b^{*}$ axes. A typical rocking curve of the $(0,0,4)$

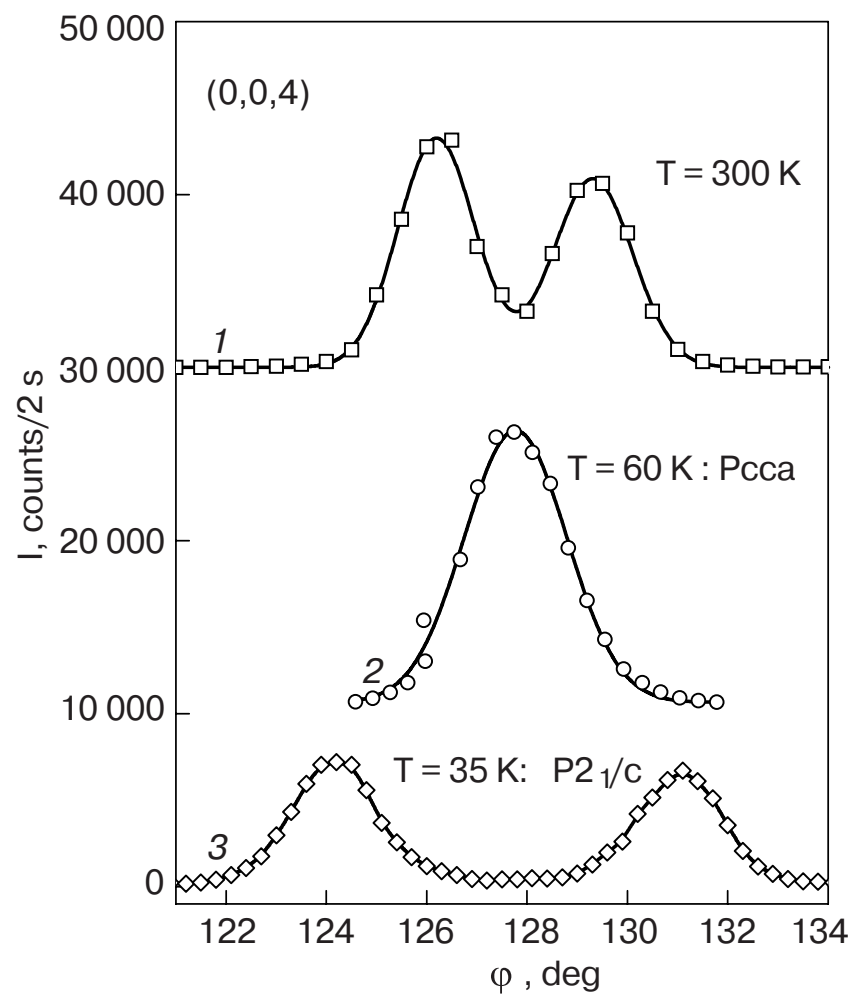

Fig. 2. Rocking curves of the $(0,0,4)$ Bragg peak (rotation around $(0,1,0)$ measured at different temperatures in a $\mathrm{CsDy}\left(\mathrm{MoO}_{4}\right)_{2}$ single crystal. The crystal structure is monoclinic at room temperature (1), becomes orthorhombic (Pcca) below $T_{1} \approx 120 \mathrm{~K}(2)$, and finally turns monoclinic again $\left(P 2_{1} / c\right)$ on cooling through $T_{2} \approx 40 \mathrm{~K}(3)$.
Bragg reflection, rotating around the $[0,1,0]$ axis, is shown in Fig. 2 and illustrates the domain structure. It should be noted that monoclinic structure at the room temperature correlates with recent $\mathrm{x}$-ray analysis [17]. No further crystallographic information was obtained at room temperature, since our main interest was with the low-temperature behavior.

Below $T_{1} \approx 120 \mathrm{~K}$, a structural phase transition takes place and $\mathrm{CsDy}\left(\mathrm{MoO}_{4}\right)_{2}$ becomes orthorhombic (Fig. 2, curve 2). A analysis of the 4-circle diffraction data suggests the space group in this low-temperature (LT-1) phase is Pcca, with the $50 \mathrm{~K}$ cell parameters $a=18.760(7) \quad \AA=2 a_{P c c m}, \quad b=5.013(4) \quad \AA \quad$ and $c=$ $=7.920(6) \AA$. Compared to the «parent» Pccm structure the $a$ axis is doubled and the Dy ions are displaced along the $c$ axis in a direction that alternates from site to site along the $a$ axis [18]. The Dy point group is $C_{2}$, as compared to $D_{2}$ in the Pccm cell.

On cooling through $T_{2} \approx 40 \mathrm{~K}$ a structural phase transition to yet another monoclinic structure occurs (Fig. 2, curve 3). It is interesting to note that both crystallographic transitions at $T_{1}$ and $T_{2}$ are totally reversible. The low-temperature monoclinic (LT-2) phase is of $P 21 / c$ symmetry, with $a=18.86(5) \AA=$ $=2 a_{P c c m}, b=10.05(3) \AA=2 b_{P c c m}, c=7.78(2) \AA$ and $\beta=86.6(2)^{\circ}$ at $T=15 \mathrm{~K}$. As in the high-temperature monoclinic phase, the two types of monoclinic domains share common $a^{*}$ and $b^{*}$ axes. Atom positions above $\left(50^{\circ} \mathrm{K}\right)$ and below $\left(15^{\circ} \mathrm{K}\right)$ phase transition at $T_{2}$ are summarized in Table 1. The phase transition at $T_{2}$ has been previously observed by Zvyagin et al. [10], but the low-temperature phase structure could not be determined from their experiments. It is accompanied by an abrupt change in the energy spectrum of the $\mathrm{Dy}^{3+}$ ions which is due to a lowering of the Dy site symmetry (point group 1 in the LT-2 phase) [19]. In the low-temperature phase there are two crystallographically inequivalent Dy sites [20].

\subsection{Magnetic long-range order}

Below $T_{N}=1.36 \mathrm{~K}$ new magnetic Bragg reflections with Miller indices $\left(P 2_{1} / c\right.$ notation $)(h, 0, l)$ ( $h$-odd, $l$-even $)$ and $(h, k, 0)(h, k$-integer, $h$-odd, $k$-even $)$ were observed in the $(0,1,0)$ and $(0,0,1)$ reciprocal-space planes, respectively. Much weaker magnetic peaks were seen at $(h, 0, l)(h, l$-odd $)$ positions. Constraints imposed by the experimental geometry prevented us from measuring magnetic scattering in other planes. Most of the diffraction data were collected at $T=0.35 \mathrm{~K}$ in the $(h, 0, l)$ zone. In general, magnetic reflections from different structural domains could always be separated, although partial overlap occurs in some cases. A typical $h$-scan along $(h, 0,2)$ measured at $T=0.35 \mathrm{~K}$ is presented Fig. 3. It shows both the nu- 
Table 1

Positions of atoms in the $\mathrm{CsDy}\left(\mathrm{MoO}_{4}\right)_{2}$ lattice.

\begin{tabular}{|c|c|c|c|c|c|}
\hline Atom & $x / a$ & $y / b$ & $z / c$ & occ. & scat. 1. \\
\hline \multicolumn{6}{|c|}{$\begin{array}{l}\mathrm{T}=50 \mathrm{~K} \\
P c c a(54) a=18.760(7) \AA, b=5.013(4) \AA, c=7.920(6) \AA, \\
\alpha=90^{\circ}, \beta=90^{\circ}, \gamma=90^{\circ}\end{array}$} \\
\hline Cs & 0.500000 & 0.030423 & 0.250000 & 0.5 & 0.542000 \\
\hline Dy & 0.250000 & 0.000000 & 0.265612 & 0.5 & 1.690000 \\
\hline Mo & 0.347931 & 0.476683 & 0.015128 & 1.0 & 0.695000 \\
\hline$O(1)$ & 0.346349 & 0.260980 & -0.160873 & 1.0 & 0.580300 \\
\hline $\mathrm{O}(2)$ & 0.281962 & 0.747241 & 0.014366 & 1.0 & 0.580300 \\
\hline $\mathrm{O}(3)$ & 0.430135 & 0.616141 & 0.018499 & 1.0 & 0.580300 \\
\hline$O(4)$ & 0.343858 & 0.259971 & 0.193318 & 1.0 & 0.580300 \\
\hline
\end{tabular}

$T=15 \mathrm{~K}$

$P 21 / c$ (14) $a=18.860(5) \AA, b=10.05(3) \AA, c=7.78(2) \AA$,

$\alpha=90^{\circ}, \beta=86^{\circ}, \gamma=90^{\circ}$

\begin{tabular}{c|c|c|c|c|c}
\hline $\mathrm{Dy}(1)$ & 0.748648 & 0.250331 & 0.305721 & 1.0 & 1.690000 \\
$\mathrm{Dy}(2)$ & 0.748415 & -0.249622 & 0.259788 & 1.0 & 1.690000 \\
$\mathrm{Cs}(1)$ & 0.505550 & 0.217766 & 0.252675 & 1.0 & 0.542000 \\
$\mathrm{Cs}(2)$ & 0.005550 & -0.217766 & 0.252675 & 1.0 & 0.542000 \\
$\mathrm{Mo}(1)$ & 0.840773 & -0.504712 & 0.040900 & 1.0 & 0.695000 \\
$\mathrm{O(11)}$ & 0.781186 & -0.377342 & 0.004581 & 1.0 & 0.580300 \\
$\mathrm{O}(12)$ & 0.923480 & -0.448149 & 0.036071 & 1.0 & 0.580300 \\
$\mathrm{O}(13)$ & 0.842379 & -0.625712 & 0.229138 & 1.0 & 0.580300 \\
$\mathrm{O(14)}$ & 0.844800 & -0.617309 & -0.105179 & 1.0 & 0.580300 \\
$\mathrm{Mo}(2)$ & 0.647130 & 0.008269 & 0.081290 & 1.0 & 0.695000 \\
$\mathrm{O}(21)$ & 0.654182 & 0.127886 & 0.245055 & 1.0 & 0.580300 \\
$\mathrm{O}(22)$ & 0.654667 & 0.117729 & -0.084289 & 1.0 & 0.580300 \\
$\mathrm{O}(23)$ & 0.717159 & -0.122156 & 0.015003 & 1.0 & 0.580300 \\
$\mathrm{O}(24)$ & 0.571327 & -0.049736 & 0.086437 & 1.0 & 0.580300 \\
$\mathrm{Mo}(3)$ & 0.649459 & -0.008883 & 0.520559 & 1.0 & 0.695000 \\
$\mathrm{O}(31)$ & 0.649830 & -0.117424 & 0.708992 & 1.0 & 0.580300 \\
$\mathrm{O}(32)$ & 0.653336 & -0.119275 & 0.347094 & 1.0 & 0.580300 \\
$\mathrm{O}(33)$ & 0.566060 & 0.068861 & 0.544750 & 1.0 & 0.580300 \\
$\mathrm{O(34)}$ & 0.715736 & 0.121061 & 0.538746 & 1.0 & 0.580300 \\
$\mathrm{Mo}(4)$ & 0.841970 & 0.509006 & 0.494863 & 1.0 & 0.695000 \\
$\mathrm{O(41)}$ & 0.846766 & 0.624122 & 0.296780 & 1.0 & 0.580300 \\
$\mathrm{O(42)}$ & 0.783495 & 0.379413 & 0.524604 & 1.0 & 0.580300 \\
$\mathrm{O(43)}$ & 0.841672 & 0.620472 & 0.663917 & 1.0 & 0.580300 \\
\hline \hline
\end{tabular}

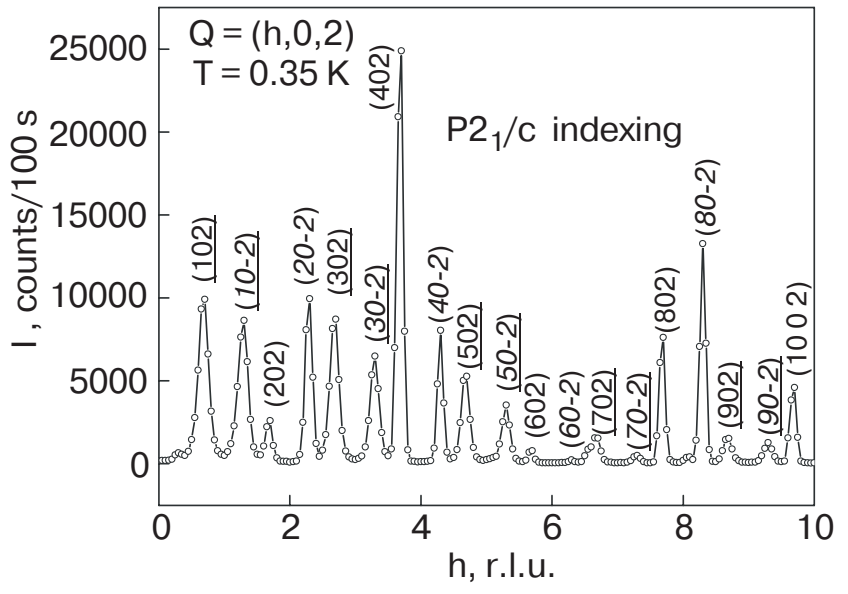

Fig. 3. Elastic scan along $(h, 0,2)_{P 2} / c$ measured in a CsDy $\left(\mathrm{MoO}_{4}\right)_{2}$ single crystal. Bragg reflections originating from the two monoclinic domains are labeled in italic and normal characters, respectively. The indices of magnetic reflections are underlined.

clear and magnetic peaks originating from the two monoclinic domains.

Peak shapes. As can be seen in Fig. 3 (for example, by comparing the $(3,0,2)$ magnetic and $(4,0,2)$ nuclear peaks) the magnetic Bragg reflections are visibly broader than the nuclear ones. This anomalously large width of magnetic reflections, more clearly seen in Fig. 4, was only observed along the $a^{*}$ direction. The $Q$-width of all the magnetic peaks investigated is resolution-limited in the $b^{*}$ and $c^{*}$ directions. The anoma-

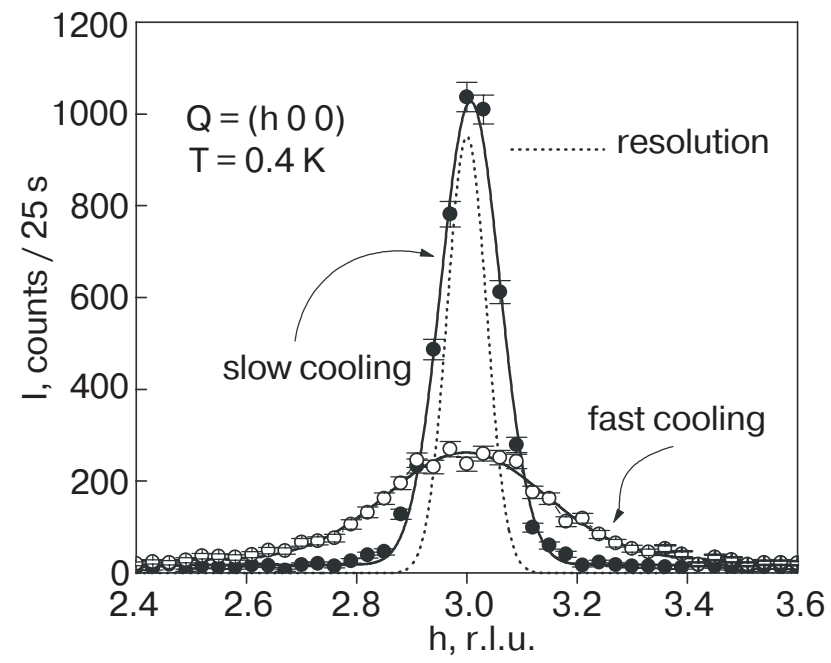

Fig. 4. Longitudinal $h$-scans through the $(3,0,0)$ magnetic Bragg peak in $\mathrm{CsDy}\left(\mathrm{MoO}_{4}\right)_{2}$ measured at $T=0.4 \mathrm{~K}$. Fast cooling through $T_{N}$ produces very broad peaks (open circles), as compared to the experimental resolution (dotted line). Slow cooling results in much sharper Bragg reflections (solid circles). The $k$ - and $l$-width of all magnetic reflections is resolution-limited independently of the cooling rate. 
lous $h$-width was found to be dependent on the rate at which the sample is brought down to low temperature. Fast cooling $(10 \mathrm{~min})$ from $T=1.5 \mathrm{~K}$ down to $T=0.35 \mathrm{~K}$ produces the broadest peaks (Fig. 4, open circles), whereas slow (2 hours) cooling through $T_{N}$ results in sharper peaks (Fig. 4, solid circles). The $Q$-integrated magnetic Bragg intensity was slightly higher in slow-cooling experiments. Moreover, some annealing (sharpening and intensifying of the magnetic peaks) over a time scale of 24 hours was observed even at $T=0.35 \mathrm{~K}$.

Order parameter. The large time constants associated with the establishing of long-range magnetic order make temperature-dependent measurements of the order parameter extremely difficult. $T_{N} \approx 1.3 \mathrm{~K}$ happens to be close to the condensation temperature of ${ }^{3} \mathrm{He}$, and long measurements above $T=1 \mathrm{~K}$ are hard to perform due to a high consumption of liquid ${ }^{3} \mathrm{He}$. The best we could do was to collect data on slow cooling (1 hour per fixed temperature with a $0.05 \mathrm{~K}$ step). The

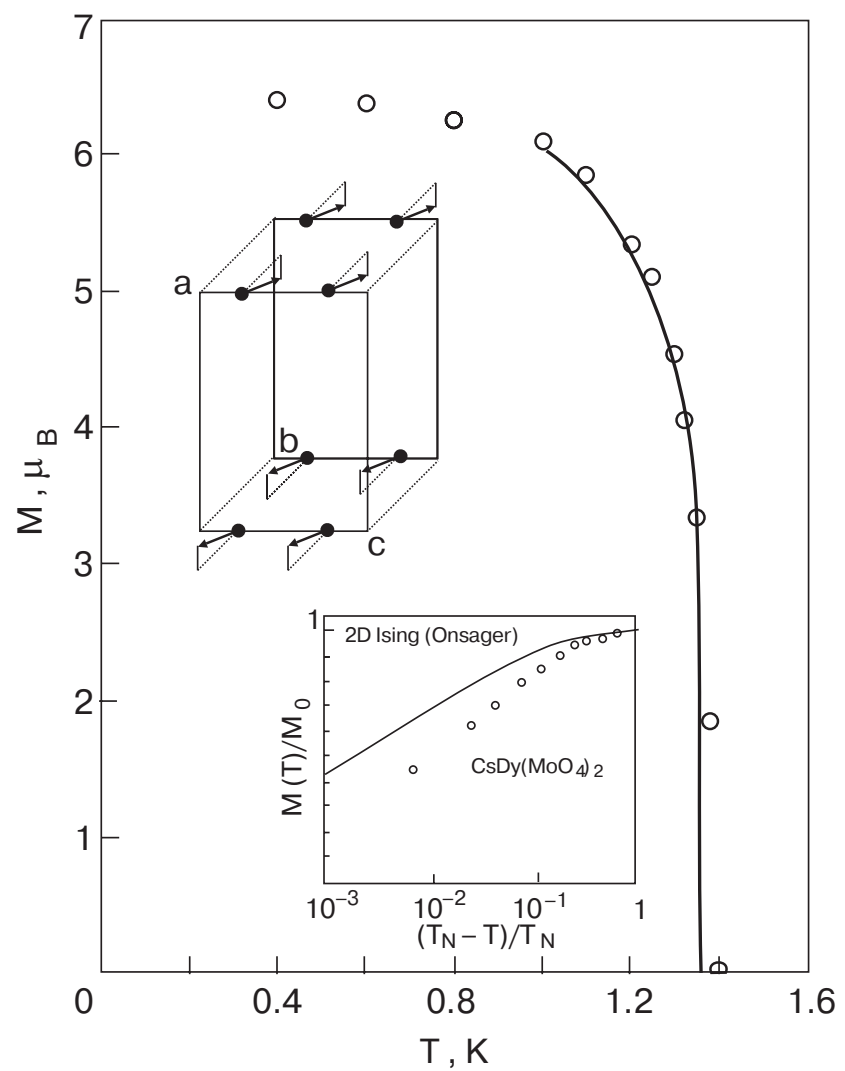

Fig. 5. Magnetic order parameter in $\mathrm{CsDy}\left(\mathrm{MoO}_{4}\right)_{2}$ plotted against temperature (circles), as deduced from the temperature dependence of the $(3,0,0)$ magnetic Bragg intensity. The solid line represents a power law with the critical exponent $\beta=0.17$ and $T_{N}=1.36 \mathrm{~K}$. The top inset shows the approximate spin structure determined at $T=0.4 \mathrm{~K}$. In the bottom inset the measured temperature dependence of the magnetization (open circles) is compared to the exact result of Onsager for a 2D Ising system (solid line). resulting order parameter deduced from the measured $(3,0,0)$ magnetic Bragg intensity is plotted against temperature in Fig. 5. The critical exponent $\beta$ and the Néel temperature were obtained in log-log plots. The analysis yielded $T_{N}=1.36(0.01) \mathrm{K}$ and $\beta=0.17(0.01)$. The normalization of the data in Fig. 5 is as discussed below.

Approximate spin structure. Detailed magnetic diffraction data could only be collected in the $(h, 0, l)$ reciprocal-space plane. Accurately determining the magnetic structure is further hindered by the necessity to make strong absorption corrections to the measured Bragg intensities. The latter introduces systematic errors, which become very important at large reflection (transmission) angles. The quantity and quality of the data do not allow us to base the analysis of magnetic intensities on the complicated low-temperature crystal structure determined in the experiments with the 4-circle diffractometer. Instead, we have utilized an $a$ priori oversimplified model for the arrangement of magnetic ions in the crystal to obtain information on the spin structure.

For each monoclinic domain the observed resolution- and absorption-corrected intensities of $P 2_{1} / c$-inequivalent $(h, 0, l)$ and $(h, 0,-l)$ Bragg peaks were found to be the same within experimental error. In addition, in our treatment we have totally neglected the $(h, 0, l)(h, l$-odd) magnetic peaks, since they are significantly weaker than those at $(h, 0, l)$ ( $h$-odd, $l$-even) and only a few could be measured. The simplified monoclinic arrangement for the $\mathrm{Dy}^{3+}$ ions that we employed in the analysis of the magnetic structure was therefore based on a reduced cell with $\tilde{a} \equiv \frac{1}{2} a_{P 21 / c} \approx 9.43 \quad \AA, \quad \tilde{b} \equiv 1 / 2 b_{P 21 / c} \approx 5.03 \quad \AA, \tilde{c} \equiv$ $\equiv c_{P 21 / c} \approx 7.82 \AA$ and $\beta=86.6(2)^{\circ}$. We have also assumed all the Dy sites to be crystallographically equivalent and positioned at $(0,0,1 / 4)$ and $(0,0,3 / 4)$. The above construct is oversimplified, but a model for the magnetic structure based on these positional coordinates accounts reasonably well for the experimental data.

A good consistency test for our experiments is an analysis of Bragg intensities measured in $h$-scans along $(h, 0,0)$. These were corrected for absorption and resolution effects and are plotted against momentum transfer in Fig. 6 (open circles). The experimental $Q$ dependence is in good agreement with a theoretical prediction for the form-factor squared of a free $\mathrm{Dy}^{3+}$ ion [21], shown as solid line in Fig. 6.

The models used for the analysis of the magnetic structure assumed a parallel alignment of nearestneighbor spins along the $b$ and $c$ axes. The nearest neighbors along $a$ were assumed to have antiparallel spins. Three models were considered, with spins lying 


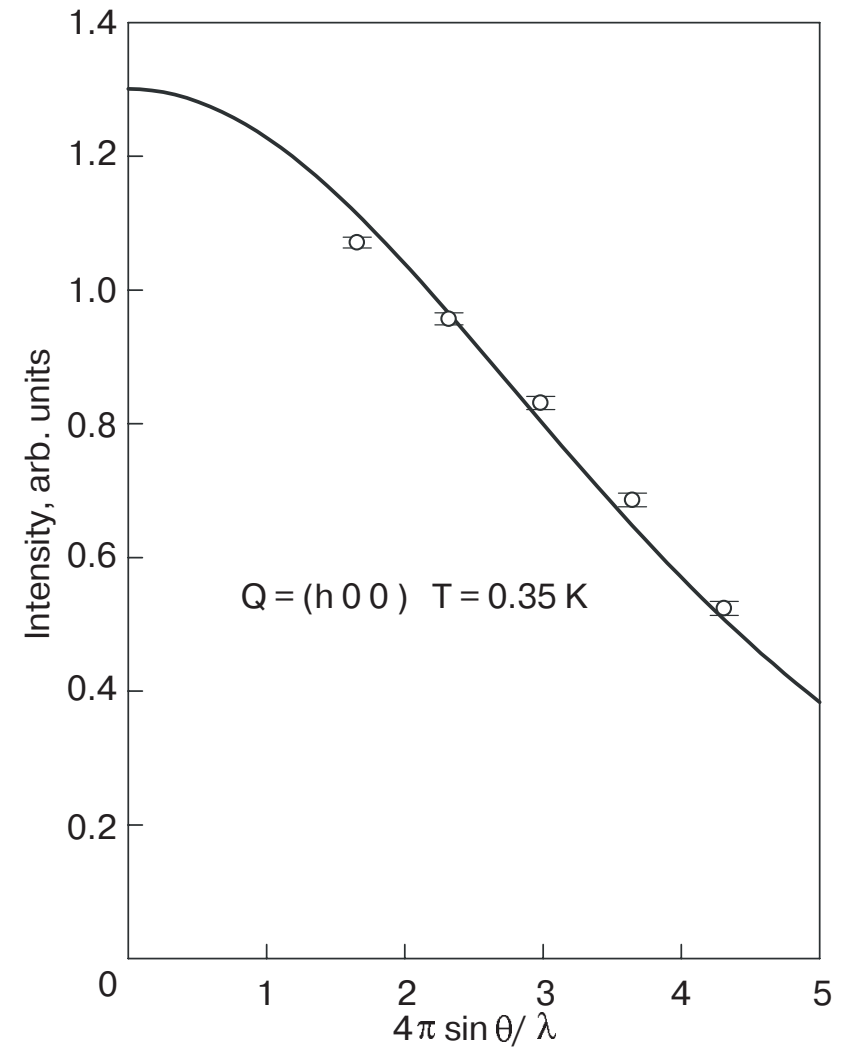

Fig. 6. Intensity of $(h, 0,0)$ magnetic Bragg reflections measured in $\operatorname{CsDy}\left(\mathrm{MoO}_{4}\right)_{2}$ as a function of momentum transfer (open circles). The data were corrected for absorption and resolution effects. The solid line represents the magnetic formfactor squared for a free $\mathrm{Dy}^{3+}$ ion.

in the $a c, b c$ and $a b$ planes. Only the last model was found to be consistent with the data. The tilt of the spins in the $a b$ plane with respect to the $b$ axis and an overall scaling factor were the only two adjustable parameters. These were refined to best fit the experimental intensities of 17 magnetic Bragg peaks of the type $(h, 0, l)$ ( $h$-odd, $l$-even) that were measured in $h$-scans to compensate for the anomalous broadening of magnetic peaks in the $a^{*}$ direction. Measurements were done separately for each monoclinic domain and corrected for resolution and absorption effects. The results of the refinement are summarized in Table 2 . Considering the limiting systematic errors arising from severe resolution and absorption effects, a reasonably good fit to the experimental data was obtained.

The refined value for the angle between the spin direction and the $b$ axis is $17.7(0.5)^{\circ}$. The resulting spin structure is visualized in the top inset in Fig. 5. The absolute value of the magnetic moment residing on the Dy sites was obtained through normalizing the magnetic Bragg intensities by those of several $(h, 0,0) \mathrm{nu}^{-}$ clear peaks. The structure factors for the latter were
List of calculated $I_{\text {calc }}$ and experimentally observed $I_{\mathrm{obs}}$ intensities for the 17 independent magnetic reflections in $\mathrm{CsDy}\left(\mathrm{MoO}_{4}\right)_{2}$ at $T=0.35 \mathrm{~K}$

\begin{tabular}{|c|c|c|c|}
\hline$(h 0 l)_{P 21 / c}$ & $I_{\mathrm{Obs}}$ & $\sigma_{o b s}$ & $I_{\mathrm{calc}}$ \\
\hline $30-2$ & 3379 & 34 & 3681 \\
\hline $50-2$ & 3092 & 49 & 3140 \\
\hline $90-2$ & 2228 & 121 & 2135 \\
\hline $110-2$ & 1720 & 110 & 1704 \\
\hline 304 & 3511 & 219 & 2656 \\
\hline 504 & 2403 & 267 & 2362 \\
\hline 704 & 2384 & 1328 & 2014 \\
\hline 102 & 4568 & 50 & 4110 \\
\hline 302 & 3726 & 56 & 3681 \\
\hline 502 & 3020 & 84 & 3140 \\
\hline 902 & 1937 & 164 & 2135 \\
\hline 1102 & 1461 & 71 & 1707 \\
\hline 500 & 3040 & 23 & 3114 \\
\hline 700 & 2716 & 25 & 2701 \\
\hline 900 & 2359 & 29 & 2254 \\
\hline 1100 & 1947 & 28 & 1819 \\
\hline 1300 & 1486 & 30 & 1427 \\
\hline
\end{tabular}

calculated from the known atomic fractional coordinates. At $T=0.35 \mathrm{~K}$ the $\mathrm{Dy}^{3+}$ moment was estimated to be $M_{\mathrm{Dy}} \approx 6.8 \mu_{B}$. This is to be compared to $M_{\text {Dy }}=g_{L} J \mu_{B} \approx 10 \mu_{B}$ for a free ion.

\subsection{Magnetic critical scattering}

Generous magnetic neutron scattering intensities allowed us to study the magnetic critical scattering, which could be plainly seen at temperatures up to $T=1.8 \mathrm{~K}$. All of the measurements were performed on the $(2.5,0,0)_{P c c m}$ magnetic Bragg reflection in a two-axis mode. Scans along $(h, 0,0)$ and $(2.5,0, l)$ were analyzed by first subtracting (point by point) the background measured at $T=4 \mathrm{~K}$ and then fitting the data to Voigt profiles. Some typical scans are shown in Fig. 7,a. The width of the Gaussian component of the Voigt function was fixed to the experimental $Q$ resolution determined from measurements on nearby nuclear Bragg reflections. The Lorentzian component represents the classical Ornstein-Zernike form for the energy-integrated neutron cross-section [22,23]:

$$
\frac{d \sigma}{d \Omega}=\frac{A T}{\kappa^{2}+q^{2}}, \quad \chi=A / \kappa^{2} .
$$


The Lorentzian widths $\kappa$ (reciprocal correlation lengths) and temperature-adjusted amplitudes $A$ were refined to best-fit the data. The solid lines in Fig. 7,a show the results of such fits. The reciprocal correlation lengths along $a^{*}$ and $c^{*}, \kappa_{a}$ and $\kappa_{c}$, respectively, are plotted against $\left(T-T_{N}\right) / T_{N}$ in Fig. $7, b$ using $T_{N}=1.36 \mathrm{~K}$. The same figure shows the temperature evolution of the staggered susceptibility $\chi$. As shown by solid lines in Fig. 7,b, the experimental temperature dependences were analyzed using power-law fits to the data. We have obtained the following values for the critical exponents: $\gamma=1.01 \pm 0.04, v_{a}=0.35 \pm$ \pm 0.04 and $v_{c}=0.94 \pm 0.07$.

\section{Discussion}

The distinguishing features of rare-earth double molybdates in general, and $\mathrm{CsDy}\left(\mathrm{MoO}_{4}\right)_{2}$ in particular, are the relatively low crystallographic symmetry and rather high magnetic moments associated with the rare-earth ions. These peculiarities make single-ion crystal-field effects extremely important and result in a huge anisotropy of magnetic susceptibility $[7,8]$. The site-symmetry of the $\mathrm{Dy}^{3+}$ ions (the ground term ${ }^{6} \mathrm{H}_{15 / 2}$ ) in the low-temperature phase is low enough for the ground state to be a single Kramers doublet with $J= \pm 15 / 2$ (Ref. 8). In other words, the $\mathrm{Dy}^{3+}$ are expected to be Ising magnetic centers. Indeed, according to EPR studies [24], there are two magnetically inequivalent sites with similar principal values of the effective $g$-factor tensor $\left(g_{a^{\prime}}=3.7 \pm 0.2, g_{b^{\prime}}=\right.$ $\left.=13.4 \pm 0.5, g_{c^{\prime}}=1 \pm 0.5\right)$. The $g$-tensor principal axis $a^{\prime}$ is reportedly tilted by $\approx \pm 10^{\circ}$ with respect to the $a$ direction in the $a b$ plane, and $b^{\prime}$ forms an angle of no more than $\approx \pm 5^{\circ}$ with the $b$ axis in the $b c$ plane. These two $g$-tensor orientations presumably correspond to the two crystallographically inequivalent $\mathrm{Dy}^{3+}$ sites that are identified in this work.

The crystal-field separation of $\mathrm{Dy}^{3+}$ Kramers doublets in $\mathrm{CsDy}\left(\mathrm{MoO}_{4}\right)_{2}$ is expected to be much larger than the ordering temperature, i.e., than the characteristic energy of magnetic interactions. The direction of magnetic moments in the ordered phase is therefore dictated by the orientations of single-ion easy axes. From our analysis of the magnetic diffraction data we conclude that the moments lie in the $a b$ crystallographic plane and are tilted by $17^{\circ}$ with respect to the $b$ axis. It is important to emphasize, though, that since two inequivalent magnetic sites are present, the actual structure should be noncollinear, and that the collinear model is no more than an approximation.

The 2D Ising character of the material becomes apparent in its critical behavior. The technique used to measure indices $v$ and $\gamma$ relies on the so-called static approximation [22], i.e., on the assumption that the
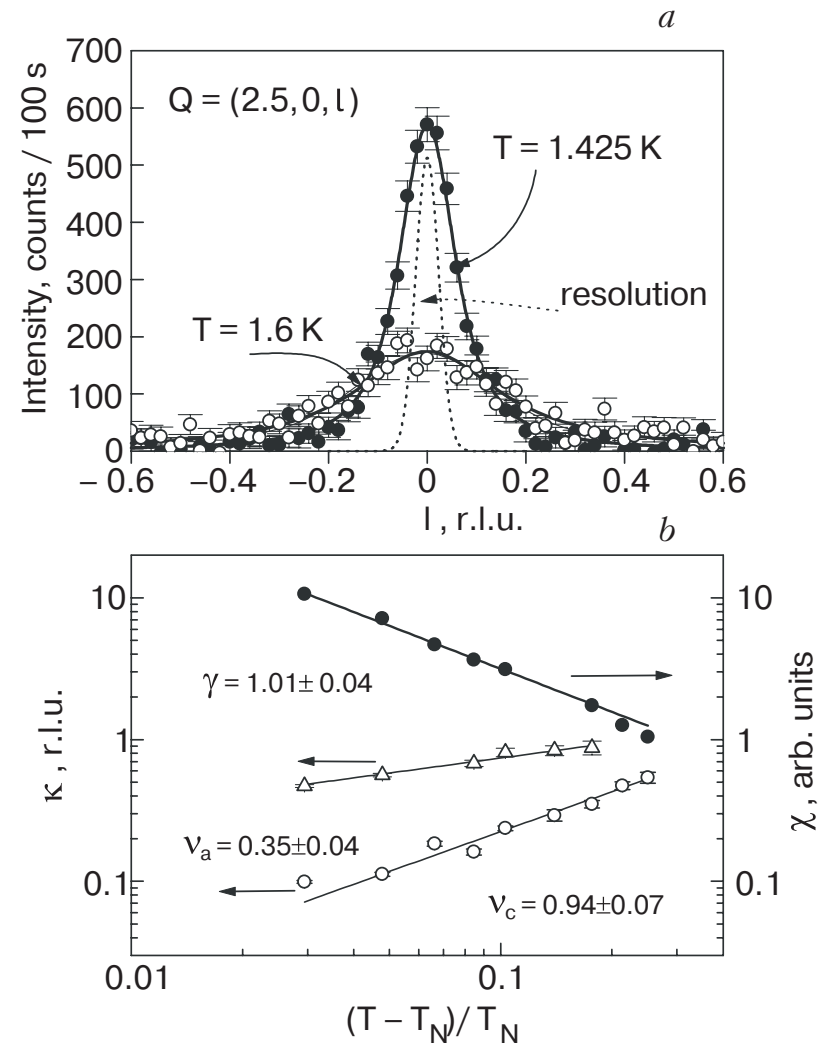

Fig. 7. Magnetic critical scattering measured in CsDy $\left(\mathrm{MoO}_{4}\right)_{2}$ above $T_{N}=1.36 \mathrm{~K}$. (a) Example two-axis scans across the magnetic zone center. The background has been subtracted, as described in the text. The solid lines represent fits with Lorentzian profiles, convolved with the Gaussian experimental resolution (dotted line). (b) Measured temperature dependence of the staggered susceptibility $\chi$ (solid circles) and reciprocal magnetic correlation length $\kappa$ along the $a$ (triangles) and $c$ (open circles) crystallographic axes. The solid lines are power-law fits to the data.

incident neutron energy in two-axis experiments is much larger than the characteristic energy width of critical scattering. Fortunately, for Ising systems the approximation is excellent, since the time scale of critical fluctuations is infinitely long. The in-plane correlation length exponent $v_{c}=0.94$, and the order-parameter exponent $\beta=0.17$ measured in $\operatorname{CsDy}\left(\mathrm{MoO}_{4}\right)_{2}$ are reasonably close to the exact characteristics of a 2D Ising magnet, where $\beta=0.125$ and $v=1$. Such behavior is consistent with the layered structure of $\mathrm{CsDy}\left(\mathrm{MoO}_{4}\right)_{2}$.

Other data obtained in the present study indicate that the temperature dependences of magnetic characteristics of $\mathrm{CsDy}\left(\mathrm{MoO}_{4}\right)_{2}$ are more complicated than simple 2D Ising behavior. In particular, the measured temperature dependence of the order parameter deviates from the exact results of Onsager in a wide temperature range (Fig. 5, bottom inset). An even more 
obvious discrepancy is revealed in the behavior of the critical index $\gamma$. The experimental value $\gamma=1$ is the same as in Ginsburg-Landau theory and quite different from that for a 2D Ising magnet, where $\gamma=1.75$ is expected. Note that in well established 2D Ising materials, such as $\mathrm{K}_{2} \mathrm{CoF}_{4}$ (Refs. 25,26) and $\mathrm{Rb}_{2} \mathrm{CoF}_{4}$ [27-29], all three critical indices $\beta, \gamma$, and $v$ are in good agreement with theory.

We tentatively propose that the main reason for any deviations from 2D Ising behavior in CsDy $\left(\mathrm{MoO}_{4}\right)_{2}$ are due to dipolar interactions. As indicated in experiments and calculations on related molybdates, dipolar coupling of Ising chains or planes may be responsible for experimentally observable short-range effects $[6,30]$, and may even drive the magnetic phase transition, as, for example, in $\mathrm{KEr}\left(\mathrm{MoO}_{4}\right)_{2}$ [5]. For $\mathrm{CsDy}\left(\mathrm{MoO}_{4}\right)_{2}$ the in-plane interactions are relatively strong and are, most likely, of exchange origin. On the other hand, interplane coupling is much weaker, and yields only short-range order between the planes. At $\left(T-T_{N}\right) / T_{N}=0.03$, for instance, the interplane magnetic correlation length amounts to only a few lattice repeats [Fig. 7,b]. All relevant interactions, but the weak interplane coupling most of all, should have a significant contribution from dipolar effects. The latter must be enhanced by i) large magnetic moments of the $\mathrm{Dy}^{3+}$ ions, and ii) ferromagnetic correlations in the planes. Indeed, the ordered moments are nearly parallel to the planes and generate a large magnetic field that favors antiparallel spin alignment in adjacent planes. If the correlations within the planes were antiferromagnetic, the dipolar field would decay much more rapidly with distance. As has been experimentally confirmed for $\mathrm{LiTbF}_{4}$ [31], dipolar magnets fall into a universality class different from that of exchange systems, and may be responsible for the observed values of critical indices in $\mathrm{CsDy}\left(\mathrm{MoO}_{4}\right)_{2}$.

We finally comment on the observed anomalous $a^{*}$-width of the magnetic Bragg reflections. The effect can be easily explained. Critical slowing down of spin fluctuations is enhanced in Ising systems. On relatively fast cooling through $T_{N}$ the short-range magnetic correlations present in the critical regime are «frozen» in the ordered phase. Since in-plane spin correlations are much stronger that interplane ones, the broadening of magnetic Bragg peaks is most pronounced in the direction normal to the planes.

\section{Conclusion}

In summary, the present investigations enabled us to determine the crystal structure of two low-temperature structural phases (below $120 \mathrm{~K}$ and below $40 \mathrm{~K}$ ), and suggest an approximate magnetic structure of the
$\operatorname{CsDy}\left(\mathrm{MoO}_{4}\right)_{2}$. A more precise model of the magnetic structure calls for further investigations.

In the ordered state the order-parameter critical exponent $\beta=0.17(0.01)$, the in-plane correlation length exponent $v=0.94(0.07)$, and the staggered susceptibility critical index $\gamma=1.01(0.04)$ were determined. Comparing these results to the exact solution for a $2 \mathrm{D}$ Ising magnet we conclude that, although $2 \mathrm{D}$ behavior is apparent in $\mathrm{CsDy}\left(\mathrm{MoO}_{4}\right)_{2}$, there are deviations from the simple 2D Ising model. One of the possible reasons is the influence of the dipole-dipole interplane interaction.

\section{Acknowledgements}

Work at ORNL and BNL was carried out under DOE Contracts No. DE-AC05-00OR22725 and DE-AC02-98CH10886, respectively.

1. P.V. Klevtsov and R.F. Klevtsova, Sov. Phys.-Crystallogr. 15, 395 (1970).

2. P.V. Klevtsov, R.F. Klevtsova, and A.V. Demenev, Sov. Phys.-Crystallogr. 17, 474 (1972).

3. V.A. Vinokurov and P.V. Klevtsov, Sov. Phys.-Crystallogr. 17, 127 (1972).

4. B.M. Wanklyan and F.R. Wonder, J. Cryst. Growth 43, 93 (1978).

5. A.G. Anders, S.V. Volotskii, and O.E. Zubkov, Fiz. Nizk. Temp. 20, 137 (1994) [Low Temp. Phys. 20, 110 (1994)].

6. A.G. Anders, S.V. Volotskii, and O.E. Zubkov, Fiz. Nizk. Temp. 20, 131 (1994) [Low Temp. Phys. 20, 105 (1994)].

7. E.N. Khatsko and A.S. Cherny, Fiz. Nizk. Temp. 11, 540 (1985) [Sov. J. Low Temp. Phys. 11, 296 (1985)].

8. E.N. Khatsko and A.S. Cherny, Ferroelectrics 130, 321 (1992)

9. E.N. Khatsko, A.S. Cherny, and A.I. Kaplienko, Fiz. Nizk. Temp. 19, 1217 (1993) [Low Temp. Phys. 19, 864 (1993)].

10. A.I. Zvyagin, S.D. El'chaninova, T.S. Stetsenko, L.N. Pelikh, E.N. Khatsko, V.I. Startsev, V.P. Soldatov, and A.I. Osetskii, Fiz. Nizk. Temp. 1, 79 (1975) [Sov. J. Low Temp. Phys. 1, 39 (1975).

11. V.I. Kut'ko, Y.N. Kharchenko, A.A. Stepanov, and N.M. Nesterenko, Fiz. Nizk. Temp. 20, 361 (1994) [Low Temp. Phys. 20, 288 (1994)].

12. A.M. Pshisukha, A.S. Cherny, and A.I. Zvyagin, Fiz. Nizk. Temp. 1, 473 (1975) [Sov. J. Low Temp. Phys. 1, 233 (1975)].

13. A.M. Pshisukha, A.I. Zvyagin, and A.S. Cherny, Fiz. Nizk. Temp. 2, 339 (1976) [Sov. J. Low Temp. Phys. 2, 170 (1976)].

14. R.F. Klevtsov and S.V. Borisov, Sov. Phys.-Doklady 12, 1095 (1968).

15. P.V. Klevtsov and L.P. Kozeeva, DAN 185, 571 (1969). 
16. R.F. Klevtsova, V.A. Vinokurov, and P.V. Klevtsov, Sov. Phys.-Crystallogr. 17, 240 (1972).

17. S.D. Elchaninova, V.P. Kuznetsov, et al., Ferroelectric 175, 85 (1996).

18. Preliminary structural refinement gives the following fractional cell coordinates for Dy in the LT-1 phase $(T=50 \mathrm{~K}):(0.25,0,0.26561)$. In the «parent» $P c c m$ structure the symmetry of the Dy site is higher: $(0.25,0,0.25)$.

19. E.N. Khatsko and A.S. Cherny, Fiz. Nizk. Temp. 7, 1048 (1981) [Sov. J. Low Temp. Phys. 7, 509 (1981)].

20. There are two inequivalent Dy sites in the LT-2 phase $(T=15 \mathrm{~K}): \quad \operatorname{Dy} 1(0.7486,0.2503,0.3057), \quad \operatorname{Dy} 2(0.7484$, $-0.2496,0.2597)$.

21. P.J. Brown, in: International Tables for Crystallography, A.J.C. Wilson (ed.), Kluwer Academic Publishers, London (1995), Vol. C, Chap. 4.4.5.

22. M.F. Collins, in: Magnetic Critical Scattering, Oxford University Press, Oxford (1989).
23. R.J. Birgeneau, J.J. Skalyo, and G. Shirane, Phys. Rev. B3, 1736 (1971).

24. A.G. Anders, V.S. Bondarenko, and A. Feher, and A. Orendacheva, Fiz. Nizk. Temp. 22, 1042 (1996) [Low Temp. Phys.22, 794 (1996)].

25. H. Ikeda and K. Hirakawa, Solid State Commun. 14,529 (1974).

26. H.R.A. Cowley, M. Hagen, and D.P. Belanger, J. Phys. C17, 3763 (1984).

27. E. Samuelsen, Phys. Rev. Lett. 31, 936 (1973).

28. H. Ikeda, M. Suzuki, and M.T. Hutchings, J. Phys. Soc. Jpn. 46, 1153 (1979).

29. M. Hagen and D.M. Paul, J. Phys. C17, 5605 (1984)

30. E.N. Khatsko, A.S. Cherny, M.I. Kobets, V.A. Pashchenko, A.I. Kaplienko, A.A. Curskas, V.V. Mitkevich, and S.M. Tret'yak, Fiz. Nizk. Temp. 20, 1022 (1994) [Low Temp. Phys. 20, 805 (1994)].

31. J. Als-Nielsen, Phys. Rev. Lett. 37, 1161 (1976). 